\title{
Disparities in breast cancer care delivery: solving a complex puzzle
}

\author{
Christopher R. Friese
}

Received: 30 January 2012/ Accepted: 21 February 2012/Published online: 8 March 2012

(C) Springer Science+Business Media, LLC. 2012

Breast cancer care delivery is known to be uneven in the United States [1]. Clinicians and researchers have long observed differences in the process and outcomes of care for women diagnosed with breast cancer that can be attributed to observable individual and societal characteristics [2-6]. Researchers have reported noteworthy differences in screening, diagnosis, treatment, and mortality by age, race, ethnicity, geographic location, education, and income. It is important to recognize there are contradictory findings in the literature, specifically regarding the relationship between race/ethnicity and quality of chemotherapy care. The seminal Institute of Medicine report Unequal Treatment recognized these differences and the broader impact of disparities in health care delivery on the nation's health [7]. The report's authors noted the complexity in both understanding and remedying the current state. A persistent challenge to correcting disparities in breast cancer care delivery is the limited understanding of the reasons for the observed disparities [8].

Currently, we have a problem of uncertain size, scope, and etiology. Research studies that include triangulated data across sources, such as cancer registries, clinical registries, and surveys of patients and providers, will help disentangle current confusion and stimulate evidence-based quality improvement and policy efforts. In this context, the paper by Lipscomb et al. in this issue of Breast Cancer Research and Treatment is a necessary piece to solve this

This is an invited commentary to article doi:

10.1007/s10549-011-1916-1

\section{R. Friese ( $\square)$}

University of Michigan School of Nursing, University

of Michigan Comprehensive Cancer Center,

400 North Ingalls \#4162, Ann Arbor, MI 48109-5482, USA

e-mail: cfriese@umich.edu complex puzzle. A key contribution from this paper is that the findings stem from a unique combination of cancer registry data and clinical records. The authors used the Georgia Comprehensive Cancer registry to identify incident cases of breast cancer and abstracted records on 868 women treated in 27 oncology settings located in relatively rural Southwest Georgia. These linked data enable us to understand, at least in part, how several personal characteristics may influence variation in high-quality breast cancer care.

The authors should be commended for their exhaustive attention to potentially confounding variables through sensitivity analyses and examination of interaction effects. Their careful attention to these issues increases the confidence in the principal study findings. In multivariable models, they found no differences in chemotherapy initiation by race. However, women who resided in impoverished areas were significantly less likely to initiate chemotherapy. This is an important finding that merits further attention and discussion. Of all the women in the study who initiated chemotherapy, $10 \%$ did not complete their originally intended plan, primarily due to toxicity. In a multivariable model that included interaction terms between race and marital status and race and comorbidity, unmarried black women had higher likelihood of chemotherapy completion compared with unmarried white women. Unmarried women with comorbid conditions were less likely to complete chemotherapy than unmarried women without comorbid conditions. Differences in chemotherapy initiation and completion were negligible for married women in the sample. One way to interpret these interesting findings is through the lens of social support.

Marital status may serve as a proxy for the larger concept of social support, which may influence treatment decisions and outcomes. While social support was not 
measured directly in this study, the presence of social support has been shown to benefit women with breast cancer $[9,10]$. Researchers have struggled to understand whether the quantity (i.e., number of people who provide support) or quality (strength of the support provided) is important [11]. A key takeaway for clinicians is to put systems in place to assess patients for both the quantity and quality of social support available to them. For patients with social support that is low in quantity, quality, or both, targeted interventions may prove beneficial. Context is also important: the authors reported that a transportation initiative, expanded insurance coverage, and other social programs were present in this community at the time of the study although it is not clear which patients participated. Strong social networks through churches or civic groups may serve as important advocates for women during treatment or help patients advocate for themselves. The expanding role of breast cancer navigators was not addressed in the study although the literature is equivocal on the efficacy of these heterogeneous programs.

Some secondary findings merit further discussion: chemotherapy initiation rates for clinically eligible patients varied significantly across the four identified treatment sites that were Commission on Cancer-accredited and the fifth category of 23 unaffiliated or free-standing practices, with a range of $21.2-47.7 \%$. Rates of planned chemotherapy completion varied significantly across practice as well (range of 83.3-100\%). This reflects the under-recognized and -studied problem of practice variation in medical oncology settings. The National Cancer Policy Board identified the absence of a robust data platform for quality data reporting and improvement as a key challenge to improved cancer care quality [12]. One wonders if the practices unaffiliated with the Commission on Cancer knew their chemotherapy initiation and completion rates for this curable disease. Without such data, how can practices implement quality improvement? Moreover, the requisite data to study cancer care quality remains far too fragmented; researchers are compelled to re-invent the wheel and forge complex partnerships to obtain necessary data for each new study. The Surveillance, Epidemiology, and End-Results program at the National Cancer Institute with the available linkage to Medicare files is an important asset to studying the problem. However, tumor registry and claims data are not sufficient to understand the complex interactions between patients and providers. Until we are able to triangulate data sources-cancer registries, clinical practice records, and surveys of providers of patients-in a reliable and robust way, the goal to measure and improve the quality of cancer care delivery in the United States will remain elusive.

The article by Lipscomb et al., contributes several pieces to our as yet-incomplete puzzle of how disparities influence the process and outcome of breast cancer therapy. First, we are able to explain in part how relationships between race/ethnicity and health care delivery may be more complicated than conventional wisdom suggests. Second, a wider view of social support may be a useful lens from which to examine disparities in future research. Finally, additional data suggest noteworthy practice-level variation in the delivery in chemotherapy care.

The findings suggest we have work ahead of us as a community of clinicians and scholars devoted to improving care for women with breast cancer. First, we must create structures that enable clinicians and researchers to share data to better understand the problem. We should measure the quantity and quality of social support that our patients have during their cancer experience. For those women who are considered vulnerable to poor outcomes, whether due to race, income, comorbidity, low social support, or other factors, we need to develop interventions that support them through their care experience. These strategies will help reduce the unwanted variation in breast cancer delivery and optimize outcomes for our patients.

Acknowledgments CRF was supported by a Pathway to Independence Award from the National Institute of Nursing Research, National Institutes of Health (R00 NR010750).

\section{References}

1. Nattinger AB (2003) Quality of care for breast cancer. Med Care 41:341-343

2. Adams SA, Butler WM, Fulton J, Heiney SP, Williams EM, Delage AF, Khang L, Hebert JR (2011) Racial disparities in breast cancer mortality in a multiethnic cohort in the Southeast. Cancer. doi:10.1002/cncr.26570

3. Griggs JJ, Culakova E, Sorbero ME, Poniewierski MS, Wolff DA, Crawford J, Dale DC, Lyman GH (2007) Social and racial differences in selection of breast cancer adjuvant chemotherapy regimens. J Clin Oncol 25:2522-2527

4. Wu X-C, Lund MJ, Kimmick GG, Richardson LC, Sabatino SA, Chen VW, Fleming ST, Morris CR, Huang B, Trentham-Dietz A, Lipscomb J (2012) Influence of race, insurance, socioeconomic status, and hospital type on receipt of guideline-concordant adjuvant systemic therapy for locoregional breast cancers. J Clin Oncol 30:142-150

5. Freedman RA, Virgo KS, He Y, Pavluck AL, Winer EP, Ward EM, Keating NL (2010) The association of race/ethnicity, insurance status, and socioeconomic factors with breast cancer care. Cancer 117:180-189

6. Krieger N, Quesenberry C Jr, Peng T, Horn-Ross P, Stewart S, Brown S, Swallen K, Guillermo T, Suh D, Alvarez-Martinez L, Ward F (1999) Social class, race/ethnicity, and incidence of breast, cervix, colon, lung, and prostate cancer among Asian, Black, Hispanic, and White residents of the San Francisco Bay Area, 1988-92 (United States). Cancer Causes Control 10: 525-537

7. Smedley BD, Stith AY, Nelson AR (2002) Unequal treatment: confronting racial and ethnic disparities in health care. National Academies Press, Washington DC 
8. Hassett MJ, Griggs JJ (2009) Disparities in breast cancer adjuvant chemotherapy: moving beyond yes or no. J Clin Oncol 27: 2120-2121

9. Sherbourne CD, Meredith LS, Rogers W, Ware JE Jr (1992) Social support and stressful life events: age differences in their effects on health-related quality of life among the chronically ill. Qual Life Res 1:235-246

10. Kroenke CH, Kubzansky LD, Schernhammer ES, Holmes MD, Kawachi I (2006) Social networks, social support, and survival after breast cancer diagnosis. J Clin Oncol 24:1105-1111
11. Gass JS, Weitzen S, Clark M, Dizon DS (2007) Defining social support systems for women with breast cancer. Am J Surg 194: 501-503

12. Hewitt ME, Simeone JV (1999) Ensuring quality cancer care. National Academies Press, Washington DC 